\title{
Meeting Abstract \\ Whole Slide Image Analysis System for Quantification of Liver Fibrosis
}

\author{
Tokiya Abe, ${ }^{1}$ Yuri Murakami, ${ }^{2}$ Masahiro Yamguchi, ${ }^{2}$ \\ Yoshiko Yamashita, ${ }^{3}$ Tomoharu Kiyuna, ${ }^{3}$ Ken Yamazaki, ${ }^{1}$ Akinori Hashiguchi, ${ }^{1}$ \\ Yutaka Yasui, ${ }^{4}$ Masayuki Kurosaki, ${ }^{4}$ Namiki Izumi, ${ }^{4}$ and Michiie Sakamoto ${ }^{1}$ \\ ${ }^{1}$ Department of Pathology, Keio University School of Medicine, 35 Shinanomachi, Shinjuku-ku, Tokyo 160-8582, Japan \\ ${ }^{2}$ Global Scientific Information and Computing Center, Tokyo Institute of Technology, 2-12-1 Ookayama, Meguro-ku, \\ Tokyo 152-8550, Japan \\ ${ }^{3}$ Medical Solutions Division, NEC Corporation, 7-1, Shiba 5-chome, Minato-ku, Tokyo 108-001, Japan \\ ${ }^{4}$ Department of Gastroenterology and Hepatology, Musashino Red Cross Hospital, 1-26-1 Kyonan-cho, Musashino, \\ Tokyo 180-8610, Japan
}

Correspondence should be addressed to Michiie Sakamoto; msakamot@z5.keio.jp

Received 2 September 2014; Accepted 2 September 2014

Copyright (C) 2014 Tokiya Abe et al. This is an open access article distributed under the Creative Commons Attribution License, which permits unrestricted use, distribution, and reproduction in any medium, provided the original work is properly cited.

\section{Background and Aims}

Histological evaluation of fibrosis after a liver biopsy is crucial for evaluating the pathology of patients with chronic liver disease. We have reported image analysis allowing quantification of liver fibrosis using Elastica van Gieson (EVG) stained whole slide images (WSIs) of liver biopsy specimens [1]. In this paper, a whole slide image analysis system for quantification of liver fibrosis was developed to apply a large number of cases in routine practice.

\section{Method}

Our system was composed of 2 steps: color correction and tissue classification. Firstly, the color correction was performed by transforming the color distribution from a target WSI into a reference WSI, where the distribution was estimated by two triangle pyramids. Next, the tissue classification was performed by using quadratic discriminant function generated from RGB signal data sets in reference WSIs. After 2 steps, the WSI pixels were classified into five classes corresponding to four tissue areas: collagen fibers, elastic fibers, nucleus, and cytoplasm and one nontissue area (i.e., glass slide). Finally, the area ratios of collagen and elastic fibers were automatically quantified.

\section{Results}

WSIs of liver biopsy specimens collected from 102 patients with hepatitis $\mathrm{C}$ were analyzed by our system [2]. The system successfully corrected the color of any WSI to that of the reference WSI and precisely extracted fine collagen and elastic fibers from portal and periportal areas (Figure 1). The averaged calculation time for WSIs with hundred-millions of pixels solution was about four minutes.

\section{Conclusions}

The whole slide image analysis system could provide quantification of liver fibrosis in biopsy specimens with different color distributions, which can display continuous value, and represent a progression of liver disease. 


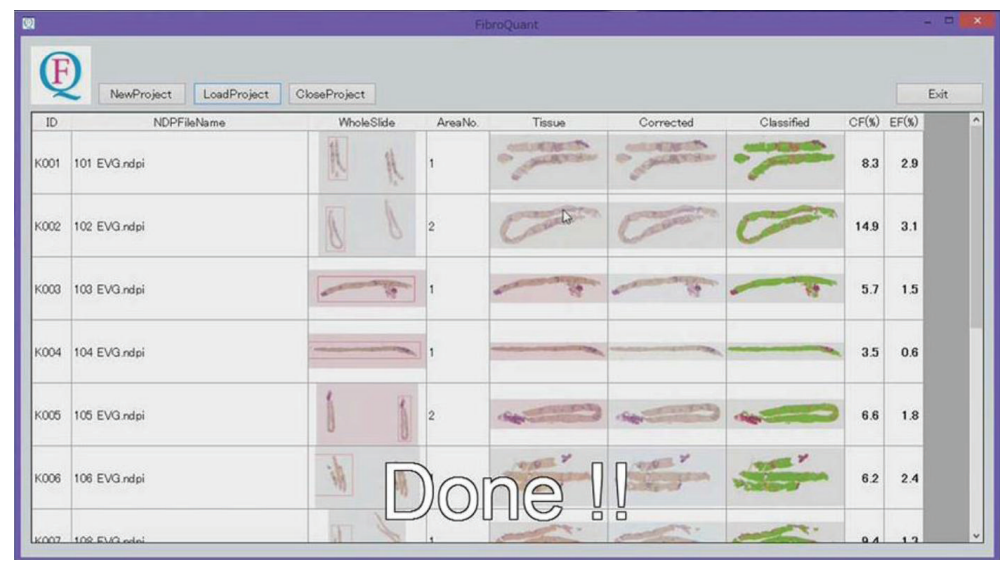

FIGURE 1: Whole slide image analysis system for quantification of liver fibrosis. The figure shows the color corrected and classified WSI and the area ratios of collagen and elastic fibers obtained by the system.

\section{Acknowledgment}

This study was supported by grants from the New Energy and Industrial Technology Development Organization (Project no. P10003).

\section{References}

[1] Y. Murakami, T. Abe, A. Hashiguchi et al., "Color correction for automatic fibrosis quantification in liver biopsy specimens," Journal of Pathology Informatics, vol. 4, p. 36, 2013.

[2] Y. Yasui, T. Abe, H. Takada et al., "Elastic fiber accumulation as a significant factor in predicting the development of hepatocellular carcinoma," in Proceedings of the International Liver Congress 49th Annual Meeting of the European Association for the Study of the Liver (EASL '14), London, UK, April 2014. 


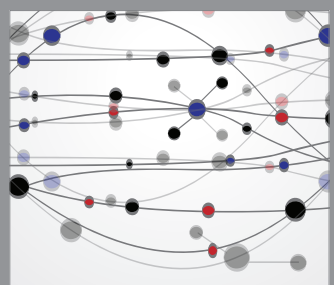

The Scientific World Journal
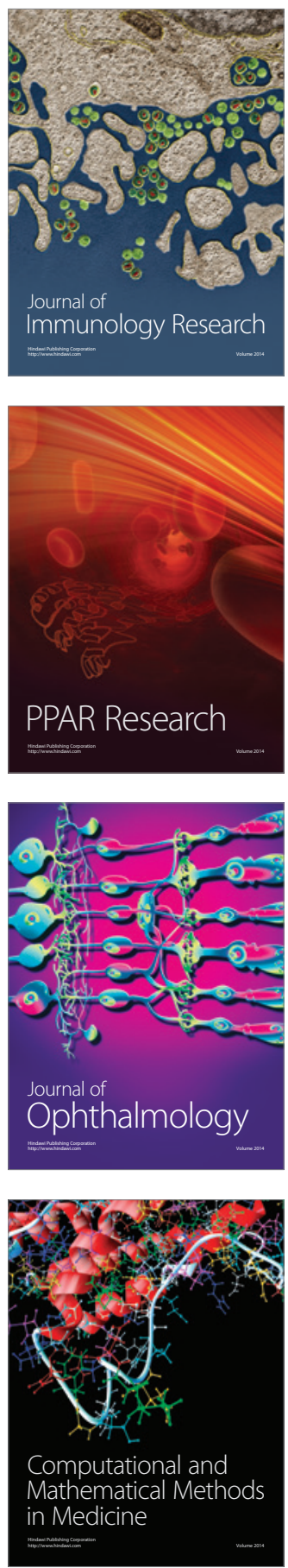

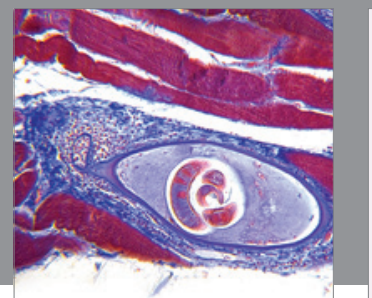

Gastroenterology

Research and Practice
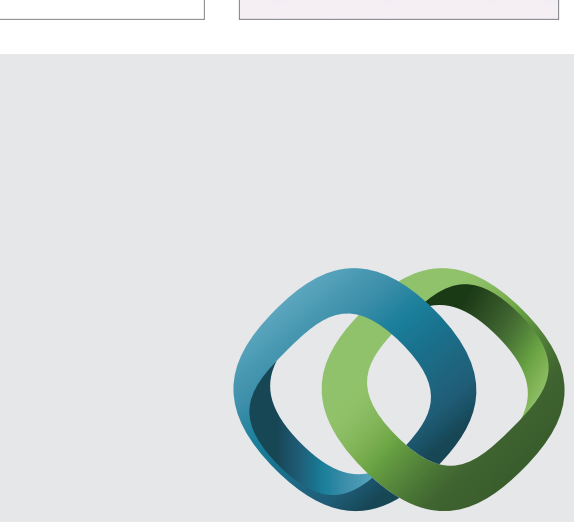

\section{Hindawi}

Submit your manuscripts at

http://www.hindawi.com
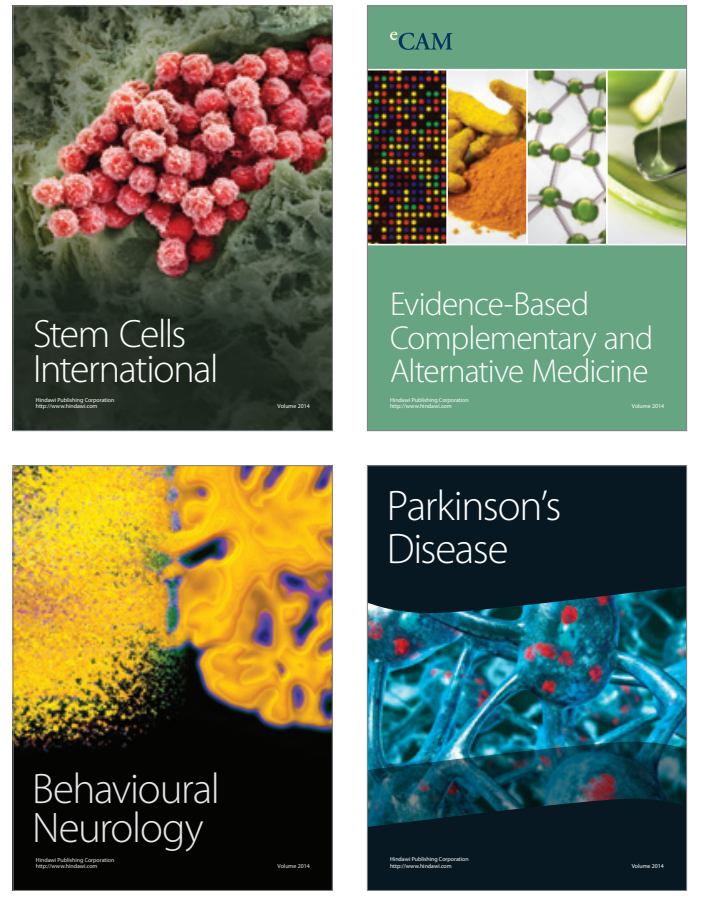
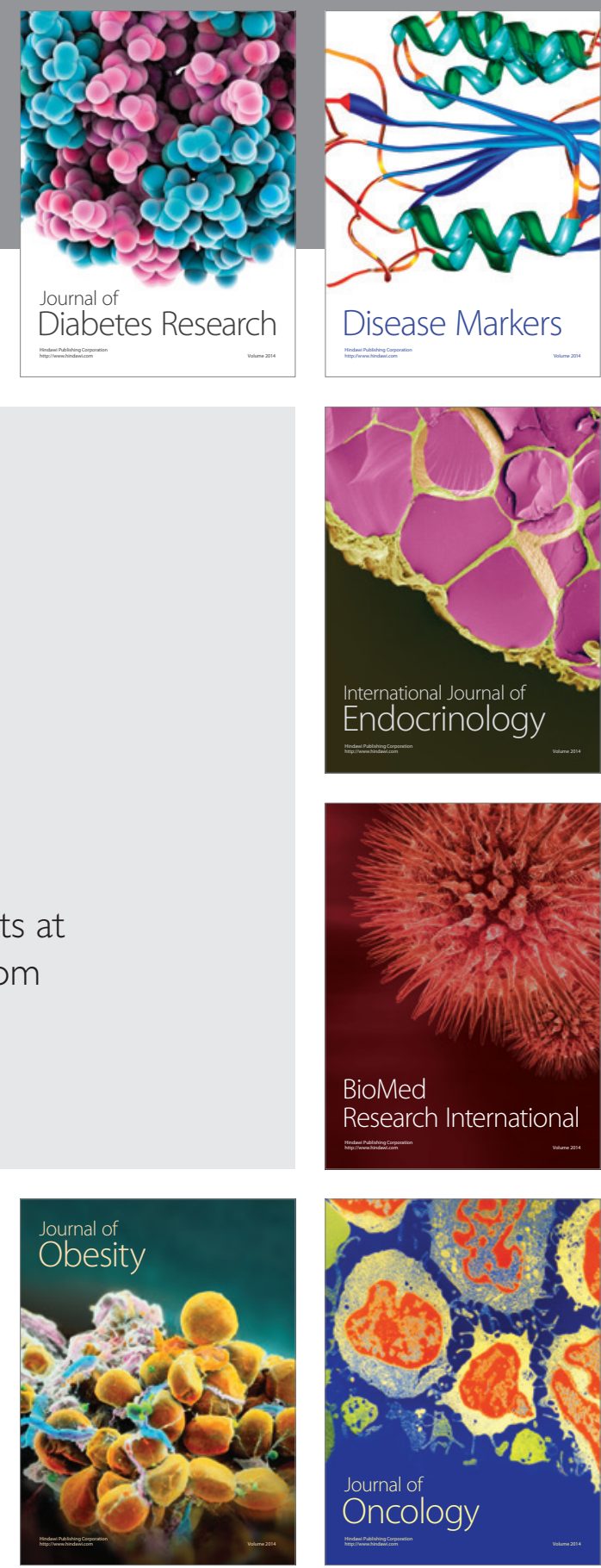

Disease Markers
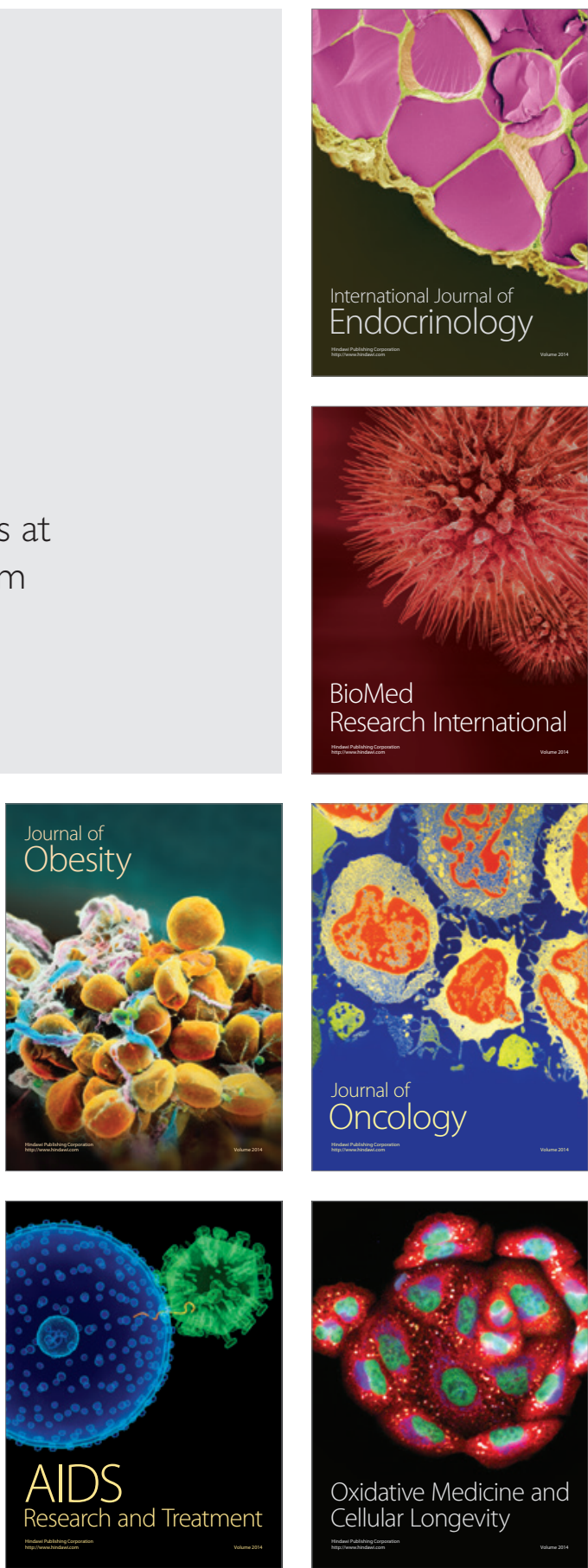